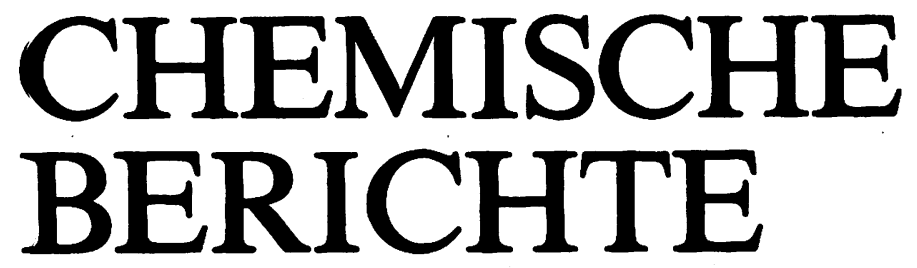

GEGRÚNDET 1868

118. JAHRGANG

HERAUSGEGEBEN IM AUFTRAG DER

GESELLSCHAFT DEUTSCHER CHEMIKER

VON

K. HAFNER - W. KIRMSE - H. MUSSO - H. NÖTH •

J. SAUER - E. WINTERFELDT

UNTER MITWIRKUNG VON

W. BECK - H. A. BRUNE - H. BUDZIKIEWICZ - W. LÜTTKE

REDAKTION: H. ZAHN

mit H. SCHILL, J. STREHLOW und A. WIELAND

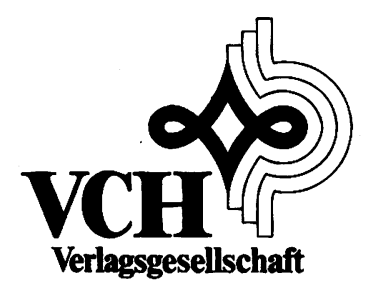




\section{INHALT VON HEFT 2}

\section{Jahrgang 1985}

\section{ANORGANISCHE CHEMIE}

Diercks Rainer und Dieck tom Heindirk: Diazadiene als Steuerliganden in der homogenen Katalyse, IX: Katalytische Cyclotetramerisierung von Propiolsäureestern ........

Beck Wolfgang, Ambach Eberhard und Nagel Ulrich: Palladium- und Platin(II)-Komplexe mit den Anionen von 6-Methyl-1,2,3-oxathiazin-4(3H)-on-2,2-dioxid und $\mathrm{N}$-2-

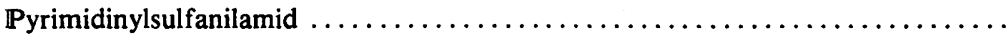

Huppmann Peter und Seppelt Konrad: Übergangsmetallverbindungen mit der Gruppe

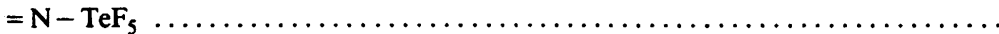

Kumpfmüller Fritz, Nölle Dieter, Nöth Heinrich, Pommerening Hans und Staudigl Rudolf: Beiträge zur Chemie des Bors, 146: Über die Reaktion von Tetrazadiborinanen mit Heterokumulenen: Pseudodipolare $[2+3]$-Cycloadditionen $\ldots \ldots \ldots \ldots \ldots$

Weber Lothar und Wewers Dietmar: Übergangsmetallkomplexe instabiler Ylide, V: Neuartige Doppelylide des Phosphors als Chelatliganden in Chromkomplexen .........

Lentz Dieter: Tetrakis(trifluormethylisocyanid)nickel, $\mathrm{Ni}\left(\mathrm{CNCF}_{3}\right)_{4}$, und $\mathrm{Bis}[\mu$-(trifluormethylisocyanid)-( $\eta$-cyclopentadienyl)nickel $],\left[N i\left(\mu-\mathrm{CNCF}_{3}\right)\left(\eta-\mathrm{C}_{5} \mathrm{H}_{5}\right)\right]_{2} \ldots \ldots \ldots$

Brink Klaus und Mattes Rainer: Mesylhydroxylamine, VI: Oxidation von $N$-Mesylhydroxylaminen - Schwingungsspektren und Kristallstruktur von 1,2-Dimesyl-1,2-di-

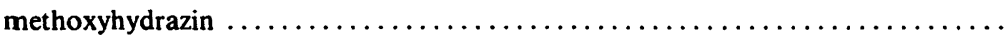

Lang Heinrich, Mohr Gerhard, Scheidsteger Olaf und Huttner Gottfried: Halogen-Metall-Austausch an komplex gebundenen Halogenphosphanen: Darstellung von

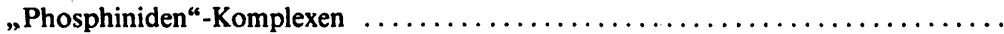

Boese Roland, Köster Roland und Yalpani Mohamed: Die Farbe von Bor-Chelaten. Röntgenstruktur-Untersuchung von Bis(4-methylphenyl)boryl- und 9-Borabicyclo[3.3.1]-

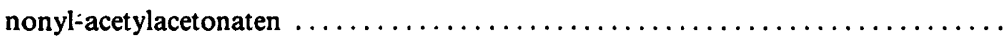

Wadepohl Hubert, Pritzkow Hans und Siebert Walter: Synthese und Struktur Platin-haltiger Tripeldecker- und Tetradecker-Komplexe $\ldots \ldots \ldots \ldots \ldots \ldots \ldots \ldots \ldots$

Appel Rolf, Knoch Falk und Zimmermann Rolf: Niederkoordinierte Phosphorverbindungen, 29: Diels-Alder-Reaktion von 2,3-Dimethylbutadien mit stabilen Phosphaalkenen als Dienophilen

\section{ORGANISCHE CHEMIE}

Lösel Walter und Daniel Helmut: 3,4-Dihydroisochinoline, I: 5,6-Dihydropyrrolo[2,1-a]isochinoline durch Umlagerung von 1-(3-Furyl)-3,4-dihydroisochinolinen . .......

Diercks Rainer und Dieck tom Heindirk: Diazadiene als Steuerliganden in der homogenen Katalyse, IX: Katalytische Cyclotetramerisierung von Propiolsäureestern ........

Wamhoff Heinrich, Wald Klemens, Kirfel Armin, Farkas Lajos, Samimi Nabi und Will Georg: Reaktionen von Uracilen, 5: Verbrückte 1,2,5,6-Tetrazocane mit Uracil- und Urazolbrücken durch Dimerisierung von 5-(1,2,4-Triazolidin-1-yl)uracilen . ....... 
Zarkadis Antonios K., Neumann Wilheln P., Marr Rainer und Uzick Wolfram: Über sterisch gehinderte freie Radikale, XII: Das Radikal tert Butyldiphenylmethyl, sein Dimeres sowie deren Autoxidation. Eine Neuuntersuchung $\ldots \ldots \ldots \ldots \ldots \ldots$

Kaupp Gerd und Knichala Bernd: Quantitative [1,3,2,3]-Eliminierung von Wasser aus

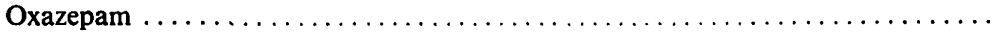

Effenberger Franz und Brodt Werner: 2(1H)-Pyridon als Austrittsgruppe bei Acylierungsreaktionen - Anwendungen in der Peptidchemie $\ldots \ldots \ldots \ldots \ldots \ldots \ldots$

Weiske Thomas, Halim Hermann und Schwarz Helmut: C-C-Spaltungen bei ionisierten Carbonsäuren als Umkehrung der [1,4]Addition von Alkyl-Radikalen an protonierte $\alpha, \beta$-ungesättigte Carbonsäuren: Zur Rolle von Enol-Radikalkationen als reaktive Zwischenstufen bei Isomerisierungs-/Dissoziationsprozessen in der Gasphase ......

Döpp Dietrich, Krüger Carl, Makedakis George und Nour-el-Din Ahmed Moukhtar: Indoleninoxide, IX: Neuartige polycyclische linear konjugierte Cyclohexadienimine durch Umlagerung instabiler Tetrahydroisoxazolo[2,3-a] indole

Quast Helmut und Nahr Uwe: Photochemische Stickstoff-Eliminierung aus 1,4-Dihydro1-phenyl-5 $\mathrm{H}$-tetrazol-5-onen und -thionen. Benzimidazolone und Carbodiimide ....

Sohár Pál, Lázár János und Bernáth Gábor: Isolierung und Strukturaufklärung eines bei der Aminomethylierungsreaktion von $\alpha$-Methylstyrol entstehenden Nebenproduktes

Siegel Herbert, Eisenhuth Ludwig und Hopf Henning: Alkine und Cumulene, XVII: Photoadditionen von Vinylacetylen an andere ungesättigte Kohlenwasserstoffe...$\ldots$.

Anke Lutz und Weyerstahl Peter: Inter- versus intramolekulare Friedel-Crafts-Reaktion von phenylsubstituierten Dichlorcyclopropanen $\ldots \ldots \ldots \ldots \ldots \ldots \ldots \ldots \ldots \ldots$

Daub Jörg, Lüdemann Hans-Dietrich, Michna Martin und Strobl Reinhard M.: Über das korrespondierende Verhalten von heteroatom-stabilisierten Carbokationen und dem Cycloheptatrien-Norcaradien-Gleichgewicht $\ldots \ldots \ldots \ldots \ldots \ldots \ldots \ldots \ldots \ldots$

Hoffmann Reinhard W. und Barth Wolfgang: Carben-Reaktionen, XVII: Nitrilylide

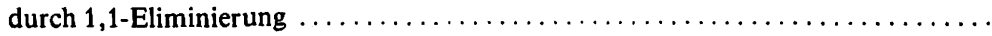

Kupfer Rainer, Würthwein Ernst-Ulrich, Nagel Michael und Allmann Rudolf: 1-Alkoxy2-azaallenium-Salze: Röntgenographische und quantenmechanische Strukturunter-

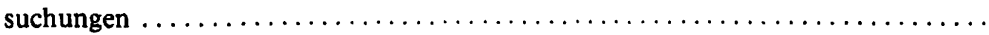

Verkoyen Carl und Rademacher Paul: Reduktion von $\beta$-Lactamen, III: Darstellung von $\beta$-Thiolactamen und Reduktion mit Raney-Nickel ....................

Yalpani Mohamed und Wilke Gilnther: Hydratisierte Oxokohlenwasserstoffe, IV: Silylierung von 1,1-Dihydroxy-Verbindungen

Boese Roland, Köster Roland und Yalpani Mohamed: Die Farbe von Bor-Chelaten. Röntgenstruktur-Untersuchung von Bis(4-methylphenyl)boryl- und 9-Borabicyclo[3.3.1]-

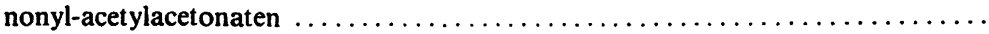

Horner Leopold und Lindel Hans: Phosphororganische Verbindungen, 111: Phosphinund Thiophosphinsäure-cyanide als fluoreszierende SH-selektive Reagenzien .....

Bäuml Englbert und Mayr Herbert: Silbertrifluoracetat-initiierte Reaktionen von Chlortriphenylallen mit Cyclopentadien - Vinylkation-analoge Cycloadditionen von Alle-

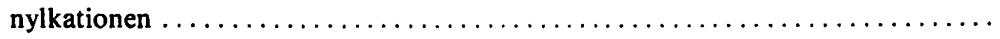


Bäuml Englbert und Mayr Herbert: Cycloadditionen des Triphenylallenyl-Kations mit Cyclopentadien - Studium des Reaktionsmechanismus unter stabilen Ionen-Bedin-

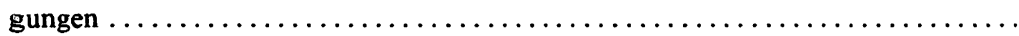

Maier Günther, Roth Cornelia und Schmitt Reinhart K.: Diastereoselektivität bei der Hydridreduktion acyclischer Diketone $(1,2-, 1,3-, 1,4-$ und 1,5 -Induktion) . . . . . . . .

Maier Günther, Schmitt Reinhart K. und Seipp Ulrich: Umkehrung der Diastereoselektivität bei der Hydridreduktion acyclischer Diketone $\ldots \ldots \ldots \ldots \ldots \ldots \ldots \ldots$

Effenberger Franz, Ziegler Thomas und Schönwälder Karl-Heinz: Enolether, XVI: Syn-

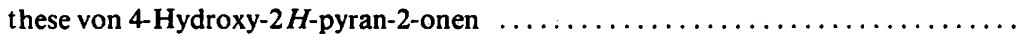

Lenoir Dieter und Frank Robert M.: Polare Effekte von 4-Substituenten bei der Solvolyse von Tetracyclo[6.2.1.1 ${ }^{3,6} \cdot 0^{2,7}$ ]dodecan-11-yl-triflaten. Through-space-Effekt im Vergleich zum induktiven Effekt

Bauer Walter, Laube Thomas und Seebach Dieter: Kristall- und Molekülstruktur eines mit THF solvatisierten dimeren Lithiumenolats eines Carbonsäureamids

Gotthardt Hans, Feist $\dagger$ Ulrich und Schoy-Tribbensee Sabine: Synthese und Eigenschaften

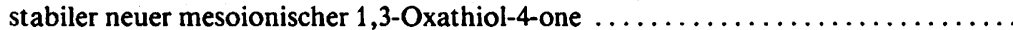

Gotthardt Hans und Feist $\uparrow$ Ulrich: Ein neuer Zugang zu Alkylthio(trifluoracetyl)furanen durch thermische $[3+2]$-Cycloadditionen neuer mesoionischer 1,3-Oxathiol-4-one an

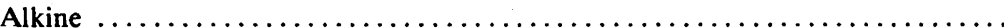

Staab Heinz A., Jörns Manfred, Krieger Claus und Rentzea Marina: Elektron-DonorAcceptor-Verbindungen, XXXVI: Chinone und Chinhydrone der [2.2]- und [3.3]Metaparacyclophan-Reihen

Eilbracht Peter, Balß Erika und Acker Michael: Zur Regio- und Stereoselektivität der hydrocarbonylierenden Cyclisierung von 1,4-Dienen mit Kohlenmonoxid: Synthese des $( \pm)-(\alpha)$-Cuparenons

Hashem Md. Abul, Hülskämper Ludwig und Weyerstahl Peter: Thermolyse und Photolyse von Hexachlor-tris- $\sigma$-homotropon und verwandten Verbindungen $\ldots \ldots \ldots \ldots$

Griesbaum Karl und Meister Martin: Beweise gegen postulierte ungewöhnlich stabile Primärozonide von 1,4-Dichlor-2-butenen $\ldots \ldots \ldots \ldots \ldots \ldots \ldots \ldots \ldots \ldots \ldots \ldots \ldots$ 


\section{INORGANIC CHEMISTRY}

Diercks Rainer and Dieck tom Heindirk: Diazadienes as Controlling Ligands in Homogeneous Catalysis, IX: Catalytic Cyclotetramerization of Propynoic Esters .......

Beck Wolfgang, Ambach Eberhard, and Nagel Ulrich: Palladium and Platinum(II) Complexes with the Anions of 6-Methyl-1,2,3-oxathiazin-4(3H)-one 2,2-Dioxide and $\mathrm{N}$ -

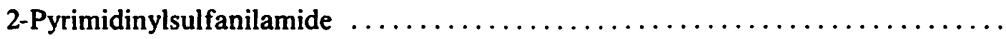

Huppmann Peter and Seppelt Konrad: Transition Metal Compounds with the Ligand

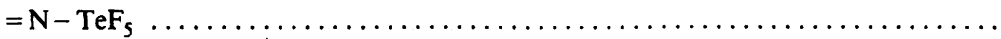

Kumpfmüller Fritz, Nölle Dieter, Nöth Heinrich, Pommerening Hans, and Staudigl Rudolf: Contributions to the Chemistry of Boron, 146: Reactions of Tetrazadiborinanes with Heterocumulenes: Pseudodipolar [2+3]-Cycloadditions

Weber Lothar and Wewers Dietmar: Transition Metal Complexes of Instable Ylides, V: Novel Double Ylides of Phosphorus as Chelating Ligands in Chromium Complexes .

Lentz Dieter: Tetrakis(trifluoromethyl isocyanide)nickel, $\mathrm{Ni}\left(\mathrm{CNCF}_{3}\right)_{4}$, and $\mathrm{Bis}[\mu$-(trifluoromethyl isocyanide)-( $\eta$-cyclopentadienyl)nickel], $\left[\mathrm{Ni}\left(\mu-\mathrm{CNCF}_{3}\right)\left(\eta-\mathrm{C}_{5} \mathrm{H}_{5}\right)\right]_{2} \ldots$

Brink Klaus and Mattes Rainer: Mesylhydroxylamines, VI: Oxidation of N-Mesylhydroxylamines - Vibrational Spectra and Structure of 1,2-Dimesyl-1,2-dimethoxy-

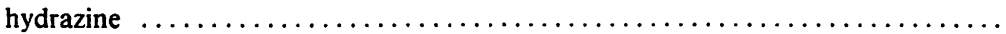

Lang Heinrich, Mohr Gerhard, Scheidsteger Olaf, and Huttner Gottfried: Halogen-Metal Exchange in Coordinated Halophosphanes: Synthesis of "Phosphinidene" Complexes

Boese Roland, Köster Roland, and Yalpani Mohamed: The Colour of Chelates of Boron. An $X$-Ray Structural Investigation of Bis(4-methylphenyl)boryl and 9-Borabicyclo-

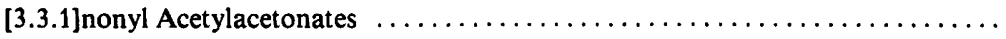

Wadepohl Hubert, Pritzkow Hans, and Siebert Walter: Synthesis and Structure of Platinum-Containing Triple-Decker and Tetra-Decker Complexes ............

Appel Rolf, Knoch Falk, and Zimmermann Rolf: Low Coordinated Phosphorus Compounds, 29: Diels-Alder Reactions of 2,3-Dimethylbutadiene with Stable Phospha-

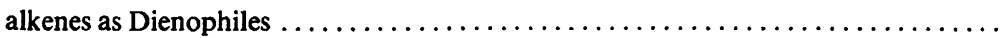

\section{ORGANIC CHEMISTRY}

Lösel Walter and Daniel Helmut: 3,4-Dihydroisoquinolines, I: 5,6-Dihydropyrrolo[2,1-a] isoquinolines via Rearrangement of 1-(3-Furyl)-3,4-dihydroisoquinolines ....

Diercks Rainer and Dieck tom Heindirk: Diazadienes as Controlling Ligands in Homogeneous Catalysis, IX: Catalytic Cyclotetramerization of Propynoic Esters ........

Wamhoff Heinrich, Wald Klemens, Kirfel Armin, Farkas Lajos, Samimi Nabi, and Will Georg: Reactions of Uracils, 5: Bridged 1,2,5,6-Tetrazocanes with Uracil and Urazole Bridges by Dimerization of 5-(1,2,4-Triazolidin-1-yl)uracils .............

Zarkadis Antonios K., Neumann Wilhelm P., Marx Rainer, and Uzick Wolfram: Sterically Hindered Free Radicals, XII: The Radical tert-Butyldiphenylmethyl, Its Dimer, and Their Autoxidation. A Reinvestigation 
Kaupp Gerd and Knichala Bernd: Quantitative [1,3,2,3]-Elimination of Water from

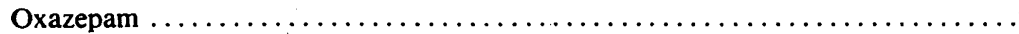

Effenberger Franz and Brodt Werner: 2(1H)-Pyridone as Leaving Group in Acylation Reactions - Applications in Peptide Synthesis $\ldots \ldots \ldots \ldots \ldots \ldots \ldots \ldots \ldots \ldots$

Weiske Thomas, Halim Hermann, and Schwarz Helmut: C-C Cleavage of Ionized Carboxylic Acids as Reversal of [1,4] Additions of Alkyl Radicals to Protonated $\alpha, \beta$ Unsaturated Acids. - On the Role of Enol Cation Radicals as Reactive Intermediates in Isomerization/Dissociation Reactions in the Gas Phase ...............

Döpp Dietrich, Krüger Carl, Makedakis George, and Nour-el-Din Ahmed Moukhtar: Indolenine Oxides, IX: Novel Polycyclic Linearly Conjugated Cyclohexadiene Imines from Rearrangement of Unstable Tetrahydroisoxazolo $[2,3-a]$ indoles $\ldots \ldots \ldots \ldots$.

Quast Helmut and Nahr Uwe: Photoextrusion of Nitrogen from 1,4-Dihydro-1-phenyl$5 \boldsymbol{H}$-tetrazol-5-ones and -thiones. Benzimidazolones and Carbodiimides ..........

Sohár Pál, Lázár János, and Bernáth Gábor: Isolation and Structure Elucidation of the By-Product Formed in the Aminomethylation of $\alpha$-Methylstyrene . . . . . . . . . .

Siegel Herbert, Eisenhuth Ludwig, and Hopf Henning: Alkynes and Cumulenes, XVII: Photoaddition of Vinylacetylene to other Unsaturated Hydrocarbons ...........

Anke Lutz and Weyerstahl Peter: Inter- versus Intramolecular Friedel-Crafts Reaction of

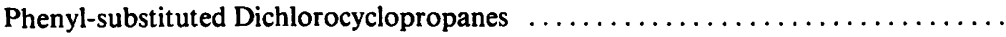

Daub Jörg, Lüdemann Hans-Dietrich, Michna Martin, and Strobl Reinhard M.: On the Correspondence of Heteroatom-stabilized Carbocations and the CycloheptatrieneNorcaradiene Equilibrium

Hoffmann Reinhard W. and Barth Wolfgang: Carbene Reactions, XVII: Nitrile Ylides via 1,1-Elimination

Kupfer Rainer, Würthwein Ernst-Ulrich, Nagel Michael, and Allmann Rudolf: 1-Alkoxy2-azaallenium Salts: $X$-Ray Crystallographic and Quantum Mechanical Structure Studies

Verkoyen Carl and Rademacher Paul: Reduction of $\beta$-Lactams, III: Synthesis of $\beta$-Thiolactams and Reduction with Raney Nickel $\ldots \ldots \ldots \ldots \ldots \ldots \ldots \ldots \ldots \ldots$

Yalpani Mohamed and Wilke Günther: Hydrated Oxocarbons, IV: Silylation of 1,1-Di-

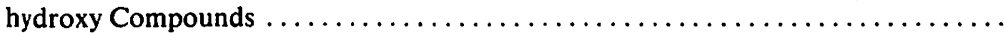

Boese Roland, Köster Roland, and Yalpani Mohamed: The Colour of Chelates of Boron. An $X$-Ray Structural Investigation of Bis(4-methylphenyl)boryl and 9-Borabicyclo[3.3.1]nonyl Acetylacetonates

Horner Leopold and Lindel Hans: Organophosphorus Compounds, 111: Phosphinic and Thiophosphinic Cyanides as Fluorescent SH-selective Reagents $\ldots \ldots \ldots \ldots \ldots \ldots$

Bäuml Englbert and Mayr Herbert: Silver Trifluoroacetate Initiated Reactions of Chlorotriphenylallene with Cyclopentadiene - Vinyl Cation Type Cycloadditions of Alle-

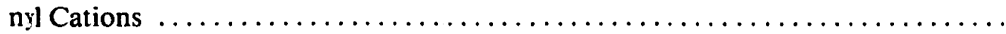

Bäumi Englbert and Mayr Herbert: Cycloadditions of the Triphenylallenyl Cation with Cyclopentadiene - Investigation of the Reaction Mechanism under Stable Ion Con-

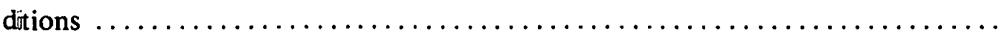

Maier Günther, Roth Cornelia, and Schmitt Reinhart K.: Stereoselectivity in the Hydride Reduction of Acyclic Diketones (1,2-, 1,3-, 1,4-, and 1,5-Induction) ............ 
Maier Günther, Schmitt Reinhart K., and Seipp Ulrich: Reversal of the Diastereoselectiv-

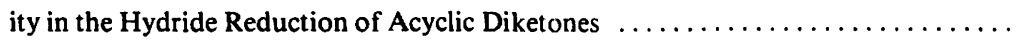

Effenberger Franz, Ziegler Thomas, and Schönwälder Karl-Heinz: Enol Ethers, XVI: Synthesis of 4-Hydroxy-2 $H$-pyran-2-ones

Lenoir Dieter and Frank Robert M.: Polar Effects of 4-Substituents in the Solvolysis of Tetracyclo[6.2.1.1 $1^{3,6} \cdot 0^{2,7}$ ]dodecan-11-yl Triflates. Through-Space vs. Inductive

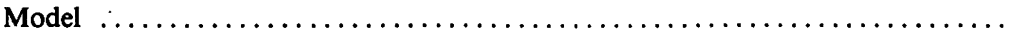

Bauer Walter, Laube Thomas, and Seebach Dieter: Crystal and Molecular Structure of a THF-solvated Lithium Amide Enolate Dimer . . . . . . . . . . . . . . . . 764

Gotthardt Hans, Feist $\dagger$ Ulrich, and Schoy-Tribbensee Sabine: Synthesis and Properties of Stable New Mesoionic 1,3-Oxathiol-4-ones $\ldots \ldots \ldots \ldots \ldots \ldots \ldots \ldots \ldots \ldots \ldots$

Gotthardt Hans and Feist + Ulrich: A New Entry to Alkylthio(trifluoroacetyl)furans by Way of Thermal [3 + 2]Cycloaddition Reactions of New Mesoionic 1,3-Oxathiol-4-

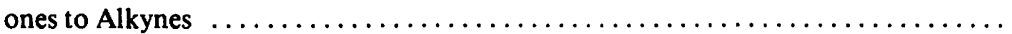

Staab Heinz A., Jörns Manfred, Krieger Claus, and Rentzea Marina: Electron DonorAcceptor Compounds, XXXVI: Quinones and Quinhydrones of the [2.2]- and [3.3]-

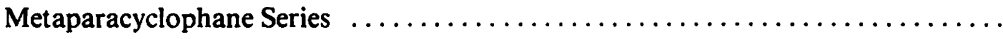

Eilbracht Peter, Balß Erika, and Acker Michael: Regio- and Stereoselectivity of the Hydrocarbonylating Cyclization of 1,4-Dienes with Carbon Monoxide: Synthesis of

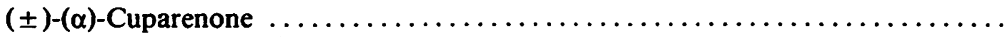

Hashem Md. Abul, Hülskämper Ludwig, and Weyerstahl Peter: Thermolysis and Photolysis of Hexachloro-tris- $\sigma$-homotropone and Related Compounds .............

Griesbaum Karl and Meister Martin: Disproval of Claimed Unusually Stable Primary Ozonides of 1,4-Dichloro-2-butenes 
Acker, M. s. Eilbracht, $P$. 825

Allmann, R. s. Kupfer, R. 643

Ambach, E. s. Beck, $W$. 444

Anke, L. und Weyerstahl, $P$. 613

Appel, R., Knoch, F. und

Zimmermann, $R$. 814

Bäuml, $E$. und Mayr, $H$. 683,694

Balß, E. s. Eilbracht, $P$. 825

Barth, W. s. Hoffmann, R. W. 634

Bauer, W., Laube, T. und Seebach, D. . . 764

Beck, W., Ambach, E. und Nagel, U. . . 444

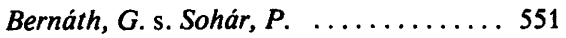

Boese, R., Köster, R. und Yalpani, M. . 670

Brink, K. und Mattes, $R$. . . . . . . . 564

Brodt, W. s. Effenberger, F. . . . . . 468

Daniel, $H$. s. Lösel, $W$. 413

Daub, J., Lüdemann, H.-D.,

Michna, M. und Strobl, R. M. . . 620

Dieck, tom, H. s. Diercks, $R$. . . . . 428

Diercks, $R$. und Dieck, tom, H. ..... 428

Döpp, D., Krüger, C., Makedakis, G.

und Nour-el-Din, A. M. . . . . . 510

Effenberger, $F$. und Brodt, $W . \ldots \ldots .468$

-, Ziegler, T. und Schönwälder, K.-H. . 741

Eilbracht, P., Balß, E. und

Acker, M. ............. 825

Eisenhuth, L. s. Siegel, H. . . . . . . . 597

Farkas, L. s. Wamhoff, H. . . . . . 436

Feist $\dagger$, U. s. Gotthardt, H. . . . 774, 785

Frank, R. M. s. Lenoir, D. . . . . . . 753

Gotthardt, $H$. und Feist,$+ U . \ldots \ldots 785$

-, Feist $†$, U. und Schoy-Tribbensee, S. . 774

Griesbaum, K. und Meister, M. . . . . 845

Halim, H. s. Weiske, T. ....... 495

Hashem, M. A., Hülskämper, L. und

Weyerstahl, P. .......... 840

Hoffmann, R. W. und Barth, W. . . . 634

Hopf, H. s. Siegel, H. ......... 597

Horner, L. und Lindel, H. . . . . . . 676

Hülskämper, L. s. Hashem, M. A. . . . . 840
Huppmann, P. und Seppelt, K. . . . . 457

Huttner, G. s. Lang, H. . . . . . . . . 574

Jörns, M. s. Staab, H. A. . . . . . . 796

Kaupp, G. und Knichala, B. ....... 462

Kirfel, A. s. Wamhoff, H. ........ 436

Knichala, B. s. Kaupp, G. . . . . . . 462

Knoch, F. s. Appel, R. .......... 814

Köster, R. s. Boese, R. . . . . . . . 670

Krieger, C. s. Staab, H. A. ........ 796

Krüger, C. s. Döpp, D. ........ 510

Kumpfmüller, F., Nölle, D., Nöth, H.,

Pommerening, $H$. und Staudigl, $R$. . 483

Kupfer, R., Würthwein, E.-U.,

Nagel, M. und Allmann, R. . . . . 643

Lang, H., Mohr, G., Scheidsteger, $O$. und Huttner, G. . . . . . . . . 574

Laube, T. s. Bauer, W. ......... 764

Lázár, J. s. Sohár, P. ............ 551

Lenoir, D. und Frank, R. M. . . . . 753

Lentz, D. . . . . . . . . . . . . . . . 560

Lindel, H. s. Horner, L. . . . . . . . 676

Lösel, $W$. und Daniel, $H . \ldots \ldots \ldots \ldots 413$

Lüdemann, H. D. s. Daub, J. . . . . . . 620

Maier, G., Roth, C. und Schmitt, R. K. . 704

—, Schmitt, R. K. und Seipp, U. . . . . 722

Makedakis, G. s. Döpp, D. ......... 510

Marx, R. s. Zarkadis, A. K. ....... 450

Mattes, R. s. Brink, K. . . . . . . . . 564

Mayr, H. s. Bäuml, E. . . . . . . . 683, 694

Meister, M. s. Griesbaum, K. ...... 845

Michna, M. s. Daub, J. . . . . . . . 620

Mohr, G. s. Lang, H. . . . . . . . . . 574

Nagel, M. s. Kupfer, R. . . . . . . . 643

Nagel, U. s. Beck, W. . . . . . . . . . 444

Nahr, U. s. Quast, H. . . . . . . . . . 526

Neumann, W. P. s. Zarkadis, A. K. . . . 450

Nölle, D. s. Kumpfmüller, $F$. . . . . 483

Nöth, H. s. Kumpfmüller, F. . . . . 483

Nour-el-Din, A. M. s. Döpp, D. ...... 510

Pommerening, H. s. Kumpfmüller, F. . . 483 
Pritzkow, H. s. Wadepohl, H. ...... 729

Quast, H. und Nahr, U. .......... 526

Rademacher, $P$. s. Verkoyen, $C$. . ... 653

Rentzea, M. s. Staab, H. A. ....... 796

Roth, C. s. Maier, G. . . . . . . . ... 704

Samimi, N. s. Wamhoff, $H . \ldots \ldots \ldots 436$

Scheidsteger, O. s. Lang, H. ....... 574

Schmitt, R. K. s. Maier, G. ...... 704, 722

Schönwälder, K.-H. s. Effenberger, $F$. . 741

Schoy-Tribbensee, S. s. Gotthardt, H. . . 774

Schwarz, H. s. Weiske, T. ........ 495

Seebach, D. s. Bauer, W. . . . . . . . . 764

Seipp, U. s. Maier, G. . ......... 722

Seppelt, K. s. Huppmann, P. ...... 457

Siebert, W. s. Wadepohl, H. . . . . ... 729

Siegel, H., Eisenhuth, L. und Hopf, H. . 597

Sohár, P., Lázár, J. und Bernáth, G. . .. 551

Staab, H. A., Jörns, M., Krieger, C. und Rentzea,..$\ldots \ldots \ldots \ldots \ldots \ldots 796$

Staudigl, R. s. Kumpfmüller, F. . . ... 483

Strobl, R. M. s. Daub, J. . . . . . . . . 6 $6 \hat{z 0}$
Uzick, W. s. Zarkadis, A. K. ....... 450

Verkoyen, $C$. und Rademacher, P. .... 653

Wadepohl, $H$., Pritzkow, $H$. und

Siebert, $W$. 729

Wald, $K$. s. Wamhoff, $\boldsymbol{H}$. 436

Wamhoff, H., Wald, K., Kirfel, A.,

Farkas, L., Samimi, N. und Will, G. 436 Weber, $L$. und Wewers, $D$. ....... 541

Weiske, T., Halim, H. und Schwarz, H. .. 495 Wewers, D. s. Weber, L. .......... 541

Weyerstahl, P. s. Anke, L. ........ 613

- s. Hashem, M. A. ........... 840

Wilke, G. s. Yalpani, M. ......... 661

Will, G. s. Wamhoff, H. ........ 436

Würthwein, E.-U. s. Kupfer, R. . . . . 643

Yalpani, M. und Wilke, G. ........ 661

- s. Boese, R. ................. 670

Zarkadis, A. K., Neumann, W. P., Marx, $R$. und Uzick, $W . \ldots \ldots \ldots 450$

Ziegler, $T$. s. Effenberger, $F$. ...... 741

Zimmermann, R. s. Appe!, R. . ..... 814 
Chem. Ber. 118, 683-693 (1985)

\title{
Silbertrifluoracetat-initiierte Reaktionen von Chlortriphenylallen mit Cyclopentadien - Vinylkation-analoge Cycloadditionen von Allenylkationen
}

\author{
Englbert Bäuml*) und Herbert Mayr *,*) \\ Institut für Organische Chemie der Universität Erlangen-Nürnberg, \\ Henkestr. 42, D-8520 Erlangen
}

Eingegangen am 6. Februar 1984

Chlortriphenylallen (8) reagiert mit Cyclopentadien in Gegenwart äquimolarer Mengen Silbertrifluoracetat zu einem Gemisch von Trifluoressigsäureestern, deren Hydrolyse die Alkohole 16-21 ergibt. Das intermediär gebildete Triphenylallenyl-Kation (9) wird hierbei ausschließlich am sp-Terminus angegriffen und geht mit Cyclopentadien Additions- sowie $[2+2]-$ und $[4+2]-$ Cycloadditionsreaktionen ein. Die experimentell beobachteten Lanthaniden-induzierten chemischen Verschiebungen der Protonen der Alkohole 17-21 stimmen gut mit den nach der McConnell-Gleichung berechneten Werten überein.

\section{Silver Trifluoroacetate Initiated Reactions of Chlorotriphenylallene with Cyclopentadiene - Vinyl Cation Type Cycloadditions of Allenyl Cations}

Chlorotriphenylallene (8) and cyclopentadiene react in the presence of equimolar amounts of silver trifluoroacetate to give a mixture of trifluoroacetates which hydrolyse to the alcohols 16-21. The intermediate triphenylallenyl cation (9) is exclusively attacked at the sp-terminus and undergoes addition as well as $[2+2]$ and $[4+2]$ cycloaddition reactions with cyclopentadiene. The experimentally observed lanthanide-induced chemical shifts of the protons of 17-21 agree well with values calculated on the basis of the McConnell equation.

Wir berichteten kürzlich, daß Allenylkationen mit Cyclopentadien in zwei grundsätzlich verschiedenen Weisen reagieren können (Schema 1) ${ }^{1)}$. Greift Cyclopentadien den $\mathrm{sp}^{2}$-Kohlenstoff des Allenylkations an, erhält man das Propargylcyclopentenyl-Kation 2 und als Folgeprodukte die Vinylkationen 1 und 3. Kommt es dagegen zum Angriff auf den sp-Terminus, entstehen das Allenylcyclopentenyl-Kation 6 oder dessen potentielle Cyclisierungsprodukte, die Allylkationen 5 und 7.

Fast ausschließlich Abfangprodukte der Kationen 1-3 erhielten wir für $R^{2}=R^{3}=$ $\mathrm{CH}_{3}, \mathrm{C}_{2} \mathrm{H}_{5}$ oder $\mathbf{R}^{2}=\mathrm{C}_{6} \mathrm{H}_{5}, \mathrm{R}^{3}=\mathbf{H}$. Nur in einigen Fällen wurden in geringen Ausbeuten Substanzen isoliert, die sich von 5-7 ableiten. Mit dem TriphenylallenylKation $\left(\mathrm{R}^{1}-\mathrm{R}^{3}=\mathrm{C}_{6} \mathrm{H}_{5}\right)$ werden dagegen ausschließlich die Reaktionswege der unteren Hälfte von Schema 1 beschritten, so daß nun die Vinylkation-analogen Reaktionsweisen von Allenylkationen ebenfalls einer systematischen Untersuchung zugänglich geworden sind ${ }^{2)}$.

*) Neue Anschrift: Institut für Chemie, Medizinische Hochschule Lübeck, Ratzeburger Allee 160, D-2400 Lübeck 1. 


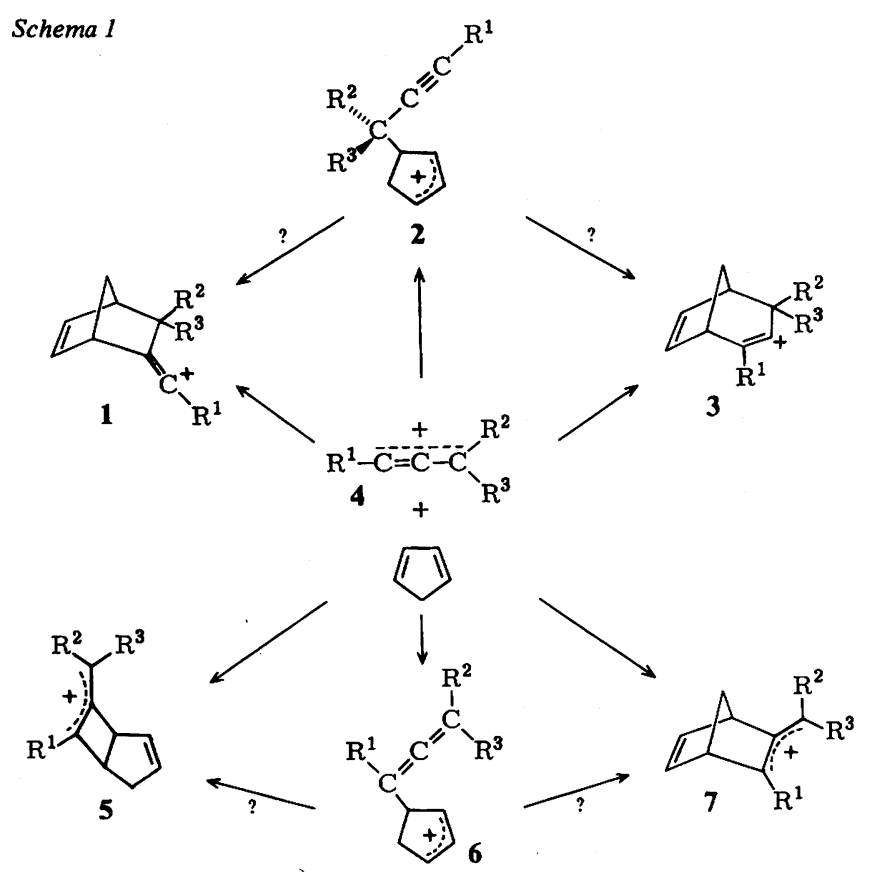

\section{Reaktionsbedingungen und Strukturaufklärung}

Beim Versuch, Chlortriphenylallen (8) in gleicher Weise ${ }^{1)}$ wie andere AllenylkationVorstufen unter Zinkchlorid-Ether-Katalyse mit Cyclopentadien umzusetzen, erhielten wir ein komplexes Produktgemisch, dessen Zusammensetzung nicht aufgeklärt wurde. Der unübersichtliche Verlauf dieser Reaktion überrascht nicht, da in Gegenwart von Zinkchlorid die Allylkationen 5-7 nicht irreversibel vom Anion abgefangen werden und somit die Möglichkeit zu Folgereaktionen haben ${ }^{3)}$.

Behandelt man dagegen 8 und Cyclopentadien mit Silbertrifluoracetat in Pentan ${ }^{4)}$, so gehen die durch Addition von 9 an Cyclopentadien entstehenden Carbenium-Ionen 12- 15 irreversible Kombinationsreaktionen mit dem Trifluoracetat-Anion ein. Nach alkalischer Aufarbeitung isoliert man das Keton 11 und die Alkohole 16-21 mit insgesamt $95 \%$ Ausbeute.

Das Keton 11 wurde durch Vergleich mit einer authentischen Probe identifiziert, die nach Meyer und Schuster durch Säure-katalysierte Umlagerung von Triphenylpropargylalkohol gewonnen wurde ${ }^{5)}$.

Die Alkohole 16 und 20 konnten durch die Übereinstimmung der ${ }^{13} \mathrm{C}$-NMR-Signale der Gerüstkohlenstoffe mit den entsprechenden Daten von 7-Isopropyliden-6-phenylbicyclo[3.2.0]hept-2-en-6-ol (22) als 6-Phenyl-7-ylidenbicyclo[3.2.0]hept-2-en-6-ole identifiziert werden (Tab. 1). Die relative Konfiguration an C-6 folgt aus den ${ }^{1} \mathrm{H}-\mathrm{NMR}$ Spektren. In 20 befinden sich $3-\mathrm{H}$ sowie $4-\mathrm{H}_{\mathrm{a}, \mathrm{b}}$ im positiven Abschirmbereich der Phenylgruppe und sind daher gegenüber $16 \mathrm{um} 0.4 \mathrm{bzw}$. etwa $0.6 \mathrm{ppm}$ nach hohem Feld verschoben. Mit dieser Zuordnung steht im Einklang, daß die sterisch abgeschirmte 
endo-OH-Gruppe in 16 wenig zur Komplexierung neigt, so daß in 16 bei Zusatz von Lanthaniden-Shift-Reagenzien nur geringe Verschiebungen auftreten (Tab. 4).

Schema 2

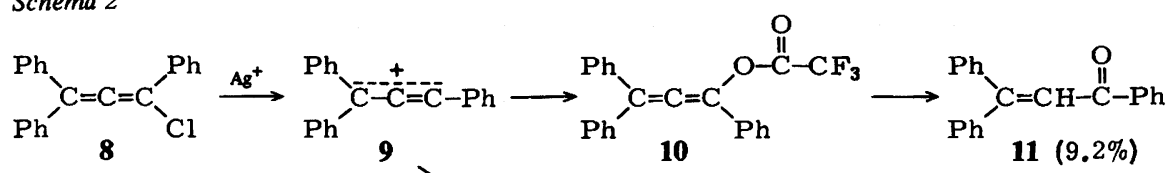

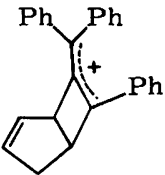

12

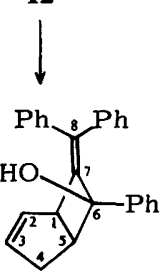

$16(6.8 \%)$

$+$

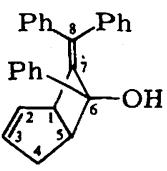

$20(10.6 \%)$<smiles>C1=C[C@H]2CC1C(=C(c1ccccc1)c1ccccc1)C2c1ccccc1</smiles>

13

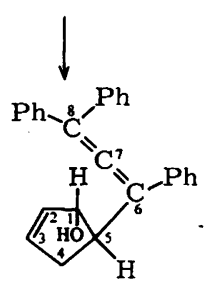

$17(22.8 \%)$

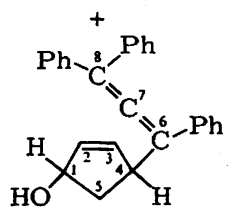

$21(14.9 \%)$
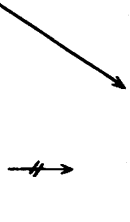<smiles>C1=CC2CC1C(=[P+](c1ccccc1)c1ccccc1)C2c1ccccc1</smiles>

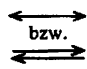<smiles></smiles>

$18(2.0 \%)$

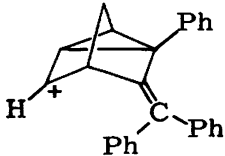

15

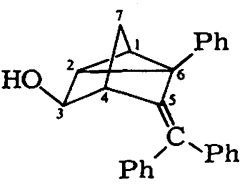

$19(28.7 \%)$

Tab. 1. ${ }^{13}$ C-NMR-Spektren einiger 7-Ylidenbicyclo[3.2.0]hept-2-en-6-ole

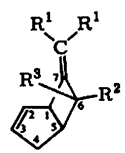

\begin{tabular}{cccccccc}
\hline $\mathrm{R}^{1}$ & $\mathrm{R}^{2}$ & $\mathrm{R}^{3}$ & Formel & $\mathrm{C}-1,5$ & $\mathrm{C}-2,3$ & $\mathrm{C}-4$ & $\mathrm{C}-6$ \\
\hline $\mathrm{CH}_{3}$ & $\mathrm{Ph}$ & $\mathrm{OH}$ & $\left.22^{\mathrm{a}}\right)$ & 48.4 & 131.6 & 31.7 & 79.8 \\
& & & & 49.5 & 132.4 & & \\
$\mathrm{Ph}$ & $\mathrm{Ph}$ & $\mathrm{OH}$ & 16 & 49.0 & 131.9 & 32.4 & 80.7 \\
& & & & 51.9 & 132.7 & & \\
$\mathrm{Ph}$ & $\mathrm{OH}$ & $\mathrm{Ph}$ & 20 & 48.7 & 130.4 & 35.3 & 84.9 \\
& & & & 51.7 & 132.8 & & \\
\hline
\end{tabular}

a) Lit. ${ }^{1)}$.

Chem. Ber. 118 (1985) 
Die Alkohole 17 und 21 (IR: $3400 \mathrm{~cm}^{-1}$ ) zeigen im IR-Spektrum keine Allenbanade, geben sich aber durch ihre ${ }^{13} \mathrm{C}$-NMR-Spektren $(17: 3 \mathrm{~s}$ bei $\delta=112.2,114.3,205.5 ;: 21$ : $3 \mathrm{~s}$ bei $\delta=112.8,114.0,205.5$ ) als tetrasubstituierte Allene zu erkennen. Außer 17 uund 21 sind somit noch die entsprechenden cis-disubstituierten Cyclopenten-Derivate dernkbar. Im ${ }^{1} \mathrm{H}$-NMR-Spektrum unterscheiden sich 17 und 21 vor allem durch die cheemische Verschiebung des zur Allengruppe geminalen Methinprotons. Wegen der dopppelten Allylstellung ist es in 21 um 0.94 ppm stärker entschirmt als in 17. Die damit moögliche Konstitutionszuordnung wird durch Entkoppeln des 1-Protons ( $\mathrm{CHOH})$ erhärttet: 1-H koppelt in 17 mit einem, in 21 hingegen mit zwei aliphatischen Protonen.

Durch Zusatz von Eu(fod) ${ }_{3}$ kann $5-\mathrm{H}$ in 17 soweit von den anderen Protonen isoliliert werden, daß es als Doppeltriplett mit einer großen (cis!) und zwei kleineren (tranns!) Kopplungen absorbiert; daraus ergibt sich die für 17 formulierte trans-Konfiguratioion. Die trans-Konfiguration von 21 folgt aus dem Befund, daß bei Zusatz von Eu(fod) ${ }_{3}$ (das Proton 4 stärker verschoben wird als das zur $\mathrm{OH}$-Gruppierung trans-ständige vicinnale 5-H. Eine relativ gute Übereinstimmung der nach der McConnell-Gleichung ${ }^{6}{ }^{\text {) }}$ bereechneten Verschiebungen mit den experimentellen Werten (Tab. 4) stützt die vorgeschlaagenen Strukturen.

18 besitzt ähnliche Strukturelemente wie das früher identifizierte 5-[(Z)- $\alpha$-Chllorbenzyliden]-endo-6-phenylbicyclo[2.2.1] hept-2-en (23), wie sich aus dem Vergleich (der ${ }^{1} \mathrm{H}$ - und ${ }^{13} \mathrm{C}$-NMR-Spektren ergibt (Tab. 2). Seine Konstitution folgt weiterhin aus ddem Befund, daß es sich durch Säure-katalysierte Umlagerung von 27 erzeugen läßt (ivgl. unten).

Tab. 2. ${ }^{1} \mathrm{H}$ - und ${ }^{13} \mathrm{C}$-NMR-Spektren zweier 5-Ylidennorbornene

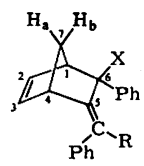

\begin{tabular}{llccccccc}
\hline $\mathrm{R}$ & $\mathrm{X}$ & Formel & $1-\mathrm{H}$ & $2-\mathrm{H}$ & $3-\mathrm{H}$ & $4-\mathrm{H}$ & $7 \mathrm{a}-\mathrm{H}$ & $7 \mathrm{~b}-\mathrm{HH}$ \\
\hline $\mathrm{Ph}$ & $\mathrm{OH}$ & $\mathbf{1 8}$ & 2.83 & 5.83 & 6.53 & 3.67 & 1.82 & 2.495 \\
$\mathrm{Cl}$ & $\mathrm{H}$ & $\mathbf{2 3}$ a) & 3.12 & 5.85 & 6.31 & 3.56 & 1.67 & 1.657 \\
\hline & & & $\mathrm{C}-1$ & $\mathrm{C}-2$ & $\mathrm{C}-3$ & $\mathrm{C}-4$ & $\mathrm{C}-6$ & $\mathrm{C}-77$ \\
\hline $\mathrm{Ph}$ & $\mathrm{OH}$ & $\mathbf{1 8}$ & 57.0 & 136.7 b) & $\left.137.0^{\mathrm{b}}\right)$ & 50.0 & 82.2 & 49.77 \\
$\mathrm{Cl}$ & $\mathrm{H}$ & $\mathbf{2 3}$ a) & 51.2 & $\left.132.8^{\mathrm{b}}\right)$ & $\left.137.0^{\mathrm{b}}\right)$ & 49.6 & 52.5 & 50.66 \\
\hline
\end{tabular}

a) Verb. $15 \mathrm{~m}$ in Lit. ${ }^{1)}$. - b) Eindeutige Zuordnung nicht möglich.

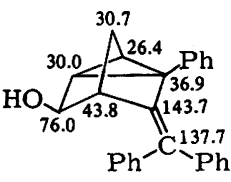

19

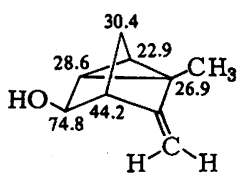

$24^{7)}$

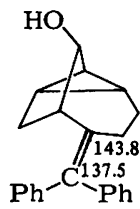

$25^{8)}$

Abb. $1 .{ }^{13} \mathrm{C}$-NMR-chemische Verschiebungen einiger tricyclischer Alkohole 
Die Struktur von 19 folgt schließlich durch Vergleich des ${ }^{13} \mathrm{C}-\mathrm{NMR}$-Spektrums mit den Signalen entsprechender Fragmente in den Tetracyclen 24 und 25. Wiederum wird die Konstitution von 19 durch die Bildung aus 27 bestätigt.

\section{Bildungsweise}

Die Entstehung der Additionsprodukte 16-21 läßt sich durch die in Schema 2 skizzierten Reaktionswege erklären. Durch Silber-Ionen-unterstützte Heterolyse von 8 entsteht zunächst das Triphenylallenyl-Kation 9, das außer mit Cyclopentadien in untergeordnetem Maß auch mit dem Trifluoracetat-Ion reagiert (9\%). Dabei bildet sich der Allenylester 10, dessen Hydrolyse das ungesättigte Keton 11 èrgibt.

Mit Cyclopentadien kann das Allenylkation entweder unter Bildung des monocyclischen Carbenium-Ions 13 reagieren oder eine $\left[2_{s}+{ }_{\pi} 2_{a}\right]$ - bzw. $\left[4_{s}+{ }_{\pi} 2_{s}\right]$-Cycloaddition eingehen, wobei die Bicyclen 12 und 14 entstehen. Da wir hier nicht entscheiden können, ob 12 und 14 stufenweise durch Cyclisierung von 13 oder durch konzertierte Cycloaddition entstehen, haben wir in Schema 2 die Formulierung gewählt, die sich aus den Versuchen unter stabilen Ionen-Bedingungen ergibt (12 stufenweise, 14 konzertiert) ${ }^{9)}$.

Vermutlich sind sterische Gründe dafür verantwortlich, daß das Allylkation 12 ausschließlich am endocyclischen Allylende angegriffen wird, so daß lediglich die beiden diastereomeren Alkohole 16 und 20 isoliert werden. Der höhere Anteil an 20 reflektiert den leichteren Zugang zur exo-Seite des bicyclischen Allylkations 12. Durch sterische Effekte ist es auch zu erklären, daß von den vier möglichen Abfangprodukten des Cyclopentenyl-Kations 13 nur zwei (17 und 21) gefunden werden. Die voluminöse Triphenylallenyl-Gruppe schirmt die Oberseite des Fünfrings so stark $\mathrm{ab}$, daß $\mathrm{CF}_{3} \mathrm{CO}_{2}^{-}$ nur von unten an den Allylteil von $\mathbf{1 3}$ herankommt.

18 ist das erwartete exo-Additionsprodukt des Norbornadienylcarbinyl-Kations 14. Da 18 aber nur mit 2\% Ausbeute isoliert wird, kann aus diesen Ergebnissen nicht auf eine Selektivität des exo-Angriffs geschlossen werden.

Formal stellt 19 ein Abfangprodukt von 15 dar. Es ist jedoch nicht klar, ob 14 und 15 im Gleichgewicht miteinander stehen oder ob sie lediglich Resonanzstrukturen derselben Spezies (Homopentadienyl-Kation) darstellen. In letzterem Fall wären 18 und 19 als Derivate einer gemeinsamen Vorstufe aufzufassen.<smiles>COC(=O)C1=C(c2ccccc2)C2C=CC1C2</smiles>

26

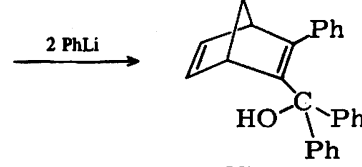

27

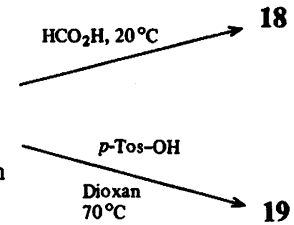

19

Unabhängig synthetisiertes 27 lagert sich unter Protonenkatalyse zunächst in 18, unter schärferen Bedingungen aber in 19 um. Dieser Befund weist auf die höhere thermodynamische Stabilität von 19 hin, erlaubt jedoch keinen Rückschluß auf die Struktur von 14 oder 15. Die Beobachtung, daß das durch Ionisation von 27 entstehende Car- 
benium-Ion 14/15 eine Vorstufe von 18 und 19 darstellt, schließt den nachstehend skkizzierten alternativen Bildungsweg ${ }^{10)}$ von 15 über das Vinylkation 28 nicht rigoros auus; sie macht ihn jedoch unwahrscheinlich, zumal Abfangprodukte des Vinylkations ; 28 nicht beobachtet wurden.

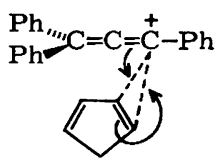

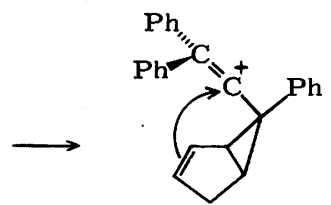

28

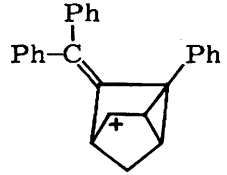

15

Der in Schema 2 vorgeschlagene Bildungsweg ist somit in der Lage, die Entstehuung aller aufgefundenen Reaktionsprodukte zu deuten. Da in Pentan, dem hier verwenadeten unpolaren Lösungsmittel, selbst relativ gut stabilisierte Carbenium-Ionen stark rmit einem Gegenion assoziiert sein sollten, erschien uns eine detailliertere mechanistisoche Studie unter diesen Bedingungen wenig aussichtsreich. Zu diesem Zweck scheinen r die in der folgenden Arbeit gewählten stabilen Ionen-Bedingungen geeigneter zu sein.

Wir danken der Deutschen Forschungsgemeinschaft und dem Fonds der Chemischen Indusstrie für die Unterstützung dieser Arbeit.

\section{Experimenteller Teil}

IR-Spektren: Beckman Acculab 1 und 3; innerhalb einer Gruppe sind die IR-Banden nach $i$ fallender Intensität geordnet. - ${ }^{1}$ H-NMR-Spektren: Jeol JNM-C-60-HL und Jeol PMX-60.. - ${ }^{13}$ C-NMR-Spektren: Jeol JNM-PS-100. - Massenspektren: Varian MAT CH 4 und Varian MAT 311 A. - Die Schmelzpunkte sind nicht korrigiert.

1-Chlor-1,3,3-triphenylpropadien (8) wurde nach Lit. ${ }^{11)}$ mit $65 \%$ Ausb. aus Triphenylprcopinol ${ }^{12)}$ und Thionylchlorid/Pyridin synthetisiert. Farblose Prismen mit Schmp. $68-69^{\circ} \mathrm{C}$ aus Ether (Lit. ${ }^{11)} 68-69^{\circ} \mathrm{C}$ ).

1. Silbertrifluoracetat-initiierte Umsetzung von 1-Chlor-1,3,3-triphenylallen (8) mit Cyvclopentadien

Eine Lơsung von $10.0 \mathrm{~g}(33.1 \mathrm{mmol}) 8$ und $6.6 \mathrm{~g}(100 \mathrm{mmol})$ Cyclopentadien in $500 \mathrm{ml} n$-Pemtan

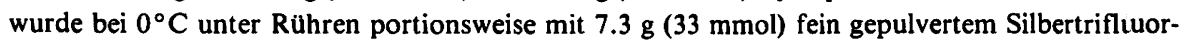
acetat versetzt. Nach 6stdg. Rühren unter Lichtausschluß wurde das abgeschiedene Silberchliorid abfiltriert und mit $50 \mathrm{ml} n$-Pentan nachgewaschen. Zur Verseifung des Esters wurde das Filltrat langsam in eine Lösung von $15 \mathrm{~g} \mathrm{(268} \mathrm{mmol)} \mathrm{KOH} \mathrm{in} 200 \mathrm{ml}$ Ethanol gegossen und $45 \mathrm{~min}$ intensiv gerührt. Nach Zusatz von $400 \mathrm{ml}$ Wasser kristallisierten noch vor der Abtrennung der wäißrigen Phase $2.34 \mathrm{~g} 19$ aus, das filtriert wurde. Nach Entfernung der wäßrigen Phase wurde die Pentanlösung auf $5^{\circ} \mathrm{C}$ gekühlt (Kühlschrank), wobei sich $2.31 \mathrm{~g}$ eines 2:1-Gemisches aus 21 uncd 19 kristallin abschieden. Nach Eindampfen der Mutterlauge im Rotationsverdampfer verblieben $7.0 \mathrm{~g}$ eines Produktgemisches, das durch Säulenchromatographie getrennt wurde $(500 \mathrm{~g}$ Kieselgel Merck, Korngröße 0.063-0.200 mm; Laufmittelgradient: 2.51 Petrolether/ $\mathrm{CH}_{2} \mathrm{Cl}_{2} 2: 1,2.51$ Petrolether $/ \mathrm{CH}_{2} \mathrm{Cl}_{2} 1: 1,2.51$ Petrolether $/ \mathrm{CH}_{2} \mathrm{Cl}_{2} 1: 2,51 \mathrm{CH}_{2} \mathrm{Cl}_{2}, 11$ Ether).

Fraktion 1: $539 \mathrm{mg}$ nicht identifizierte Produkte; Fraktion 2: $760 \mathrm{mg} \mathrm{16}$; Fraktion 3: $48 \mathrm{mgg} 16$ und 18 (1:1); Fraktion 4: $164 \mathrm{mg} \mathrm{18;} \mathrm{Fraktion} \mathrm{5:} 294 \mathrm{mg} 18$ und 11 (15: 85); Fraktion 6: $1365 \mathrm{mg}$ 
11 und 20 (45: 55); Fraktion 7: $480 \mathrm{mg} \mathrm{20;} \mathrm{Fraktion} \mathrm{8:} 1580 \mathrm{mg}$ 17; Fraktion 9: $1608 \mathrm{mg}$ eines Gemisches aus 17, 19 und 21.

Fraktion 9 wurde mit dem vor der chromatographischen Trennung isolierten Kristallgemisch von 21 und 19 vereinigt und an Kieselgel $\left(\mathrm{CH}_{2} \mathrm{Cl}_{2} /\right.$ Petrolether/Essigester 20:1:1) getrennt. Mit zunehmender Retentionszeit wurden isoliert: $1062 \mathrm{mg} \mathrm{17,} 986 \mathrm{mg} 19$ und $1728 \mathrm{mg} 21$.

Summiert man die aus den einzelnen Arbeitsgängen gewonnenen Ausbeuten, so ergeben sich $864 \mathrm{mg}(9.2 \%) 11,784 \mathrm{mg}(6.8 \%) 16,2.64 \mathrm{~g}(22.8 \%) 17,232 \mathrm{mg}(2.0 \%) 18,3.33 \mathrm{~g}(28.7 \%)$ 19, $1.23 \mathrm{~g}(10.6 \%) 20,1.73 \mathrm{~g}(14.9 \%) 21$.

7-1(Diphenylmethylen)-6-phenylbicyclo[3.2.0]hept-2-en-endo-6-ol (16): Farblose Prismen mit Schmp. 102.5 - $104^{\circ} \mathrm{C}$ (Ether). - IR (KBr): $3540(\mathrm{OH}), 2910,3040,3010(\mathrm{CH}), 1490,1595$ (Aromatengerüst), 690, 1440, 740, 720, 770, 1180, 1170, $1055 \mathrm{~cm}^{-1} .-{ }^{1} \mathrm{H}-\mathrm{NMR}\left(\mathrm{CDCl}_{3}\right): \delta=2.42$ $(\mathrm{s} ; 1 \mathrm{H}, \mathrm{OH}), 2.33-3.00(\mathrm{~m} ; 2 \mathrm{H}, 4 \mathrm{a}-\mathrm{H}, 4 \mathrm{~b}-\mathrm{H}), 3.22\left(\mathrm{td}, J_{5,1}=J_{5,4 \text {-cis }}=8.0 \mathrm{~Hz}, J_{5,4 \text {-trans }}=\right.$ $2.0 \mathrm{~Hz} ; 1 \mathrm{H}, 5-\mathrm{H}), 4.24(\mathrm{mc} ; 1 \mathrm{H}, 1-\mathrm{H}), 5.60-6.00(\mathrm{~m} ; 2 \mathrm{H}, 2-\mathrm{H}, 3-\mathrm{H}), 7.14,7.33(2 \mathrm{mc} ; 15 \mathrm{H}$, Aromaten-H). $-{ }^{13} \mathrm{C}-\mathrm{NMR}\left(\mathrm{CDCl}_{3}\right): \delta=32.4$ (t; C-4), 49.0, 51.9 (2 d; C-1, C-5), 80.7 (s; C-6), 124.7, 126.3, 126.7, 127.5, 127.7, 128.2, 128.7, 129.4 (Phenyl-C), 131.9, 132.7 (2 d; C-2, C-3), $137.4,138.3,140.2,145.2,147.7\left(5 \mathrm{~s} ; 3 \mathrm{C}_{\mathrm{ipso}}, \mathrm{C}-7, \mathrm{C}-8\right) .-\mathrm{MS}(70 \mathrm{eV}): m / e=350\left(100 \%, \mathrm{M}^{+}\right)$, 284 ((18), 273 (11), $245(51), 206(34), 178(33), 167$ (37), 111 (43), $105(73), 77$ (53), 69 (71), 67 (61), 55 (69).

trans-5-(Triphenylpropadienyl)-2-cyclopenten-1-ol (17): Farbloses, mikrokristallines Material vom Schmp. $132.0-132.5^{\circ} \mathrm{C}$ (Ether). - IR (KBr): 3520, $3400(\mathrm{OH}), 3030,2890(\mathrm{CH}), 1490$, 1595 (Aromatengerüst), 695, 770, 1020, $1445 \mathrm{~cm}^{-1}$. $-{ }^{1} \mathrm{H}-\mathrm{NMR}\left(\mathrm{CDCl}_{3}\right): \delta=1.92$ (br. s; $1 \mathrm{H}$, $\mathrm{OH}), 2.12-3.05$ (m; 2 H, 4a-H, 4b-H), 3.33 (mc; $1 \mathrm{H}, 5-\mathrm{H}), 4.95$ (br. s; $1 \mathrm{H}, 1-\mathrm{H}), 5.67-6.08$ (m; $2 \mathrm{H}, 2-\mathrm{H}, 3-\mathrm{H}), 7.17-7.77$ mit Maximum bei 7.37 (m; $15 \mathrm{H}$, Aromaten-H). $-{ }^{13} \mathrm{C}-\mathrm{NMR}$ $\left(\mathrm{CDCl}_{3}\right): \delta=38.2$ (t; C-4), 47.4 (d; C-5), 82.7 (d; C-1), 112.2, 114.3 (2 s; C-6, C-8), 126.6, 127.3, 128.2, 128.4, 128.6 (Aromaten-C), 132.4, 133.9 (2 d; C-2, C-3), 135.7 (s; C ipso), 136.4 (s; $2 \mathrm{C}_{\mathrm{ipso}}$ ), 205.5 (s; C-7). - MS (70 eV): $m / e=350\left(40 \%, \mathrm{M}^{+}\right), 269$ (92), 268 (82), 267 (90), 191 (90), 167 (100), 165 (77).

3-(Diphenylmethylen)-2-phenylbicyclo[2.2.1]hept-5-en-exo-2-ol (18): Farblose Rhomboeder vom Schmp. $107.5-108.5^{\circ} \mathrm{C}$ (Ether). - IR (KBr): 3420, $3550(\mathrm{OH}), 2970,3040,3000,2920$ $(\mathrm{CH}), 1630,1640(\mathrm{C}=\mathrm{C}), 1490,1595$ (Aromatengerüst), 695, 725, 1020, 750, 1030, $1440 \mathrm{~cm}^{-1}$. ${ }^{1} \mathrm{H}-\mathrm{NMR}\left(\mathrm{CDCl}_{3}\right): \delta=1.82(\mathrm{~d}, J=8.8 \mathrm{~Hz} ; 1 \mathrm{H}, 7 \mathrm{a}-\mathrm{H}), 2.45(\mathrm{~d}, J=8.8 \mathrm{~Hz} ; 1 \mathrm{H}, 7 \mathrm{~b}-\mathrm{H}), 2.53$ (br. s; $1 \mathrm{H}, \mathrm{OH}$ ), 2.83 (br. s; $1 \mathrm{H}, 1-\mathrm{H}), 3.67$ (br. s; $1 \mathrm{H}, 4 \mathrm{H}), 5.83$ (dd, $J_{5,6}=5.6 \mathrm{~Hz}, J_{1,6}=$ $2.8 \mathrm{~Hz} ; 1 \mathrm{H}, 6-\mathrm{H}), 6.53\left(\mathrm{dd}, J_{5,6}=5.6 \mathrm{~Hz}, J_{5,4}=2.8 \mathrm{~Hz} ; 1 \mathrm{H}, 5-\mathrm{H}\right), 7.02,7.31(2 \mathrm{mc} ; 15 \mathrm{H}$, Aromaten-H), $-{ }^{13} \mathrm{C}-\mathrm{NMR}\left(\mathrm{CDCl}_{3}\right): \delta=49.7(\mathrm{t} ; \mathrm{C}-7), 50.0(\mathrm{~d} ; \mathrm{C}-4), 57.0(\mathrm{~d} ; \mathrm{C}-1), 82.2(\mathrm{~s} ; \mathrm{C}-2)$, 126.2, 126.5, 126.7, 126.9, 127.8, 128.0, 128.7, 129.5 (Aromaten-C), 136.7, 137.0 (2 d; C-5, $\mathrm{C}-6), 136.0,141.1,142.6,145.7,146.9\left(5 \mathrm{~s} ; 3 \mathrm{C}_{\mathrm{ipso}}, \mathrm{C}-3, \mathrm{C}-8\right) .-\mathrm{MS}(70 \mathrm{eV}): \mathrm{m} / \mathrm{e}=350(100 \%$, $\mathrm{M}^{+}$), 285 (16), 284 (72), 283 (36), 245 (17), 207 (27), 206 (37), 178 (32), 167 (18), 115 (48), 105 (67).

5-(Diphenylmethylen)-6-phenyltricyclo[2.2.1.0 $0^{2.6}$ Jheptan-exo-3-ol (19): Watteartige Kristalle vom Schmp. $172-173^{\circ} \mathrm{C}\left(\mathrm{CCl}_{4} /\right.$ Pentan). - IR (KBr): $3300(\mathrm{OH}), 3040,3010,2920,2900,2860$ $(\mathrm{CH}), 1640(\mathrm{C}=\mathrm{C}), 1485,1590$ (Aromatengerüst), 1060, 1030, 885, 760, 710, 1435, $800 \mathrm{~cm}^{-1}$. ${ }^{1} \mathrm{H}-\mathrm{NMR}\left(\mathrm{CDCl}_{3}\right.$ ): $\delta=1.72$ (br. s; $1 \mathrm{H}, \mathrm{OH}$ ), 2.05 (br. s; 4 H, 1-H, 2-H, 7a-H, 7b-H), 2.70 (br. s; $1 \mathrm{H}, 4-\mathrm{H}), 4.32$ (br. s; $1 \mathrm{H}, 3-\mathrm{H}), 6.78(\mathrm{~s} ; 5 \mathrm{H}$, Aromaten-H), 6.91 (s; $5 \mathrm{H}$, Aromaten-H), 7.23 (mc; $5 \mathrm{H}$, Aromaten-H). $-{ }^{13} \mathrm{C}-\mathrm{NMR}\left(\mathrm{CDCl}_{3}\right): \delta=26.4(\mathrm{~d} ; \mathrm{C}-1), 30.0(\mathrm{~d} ; \mathrm{C}-2), 30.7(\mathrm{t} ; \mathrm{C}-7), 36.9(\mathrm{~s}$; C-6), 43.8 (d; C-4), 76.0 (d; C-3), 125.5, 125.6, 126.3, 126.6, 127.1, 127.7, 129.7, 129.9, 130.2 (Phenyl), 132.0, 139.4, 143.3 (3 s; C $m / e=350\left(100 \%, \mathrm{M}^{+}\right), 332(6), 167(53), 165(20), 91(22)$.

Chem. Ber. $118(1985)$ 
7-(Diphenylmethylen)-6-phenylbicyclo[3.2.0]hept-2-en-exo-6-ol (20): Gelbes Harz, das inicht zur Kristallisation gebracht werden konnte. - IR (KBr): $3400(\mathrm{OH}), 3030,2900(\mathrm{CH}), 1650$ $(C=C), 1595,1490$ (Aromatengerüst), 695, 760, 1440,1065, 1030,1185 $\mathrm{cm}^{-1}$. - ${ }^{1} \mathrm{H}-\mathrm{MNMR}$ $\left(\mathrm{CDCl}_{3}\right): \delta=1.43-2.67(\mathrm{~m} ; 3 \mathrm{H}, 4 \mathrm{a}-\mathrm{H}, 4 \mathrm{~b}-\mathrm{H}, \mathrm{OH}), 3.20\left(\mathrm{td}, J_{5,1}=J_{5,4 \text { cis }}=8.0 \mathrm{~Hz} ; J_{5,4 \text { traans }}=\right.$ $1.6 \mathrm{~Hz} ; 1 \mathrm{H}, 5-\mathrm{H}), 4.25(\mathrm{mc} ; 1 \mathrm{H}, 1-\mathrm{H}), 5.36-5.60$ und $5.67-5.93(2 \mathrm{~m} ; 2 \mathrm{H}$, Viny,l-H), 6.93-7.63 mit Maxima bei 7.11 und $7.35\left(\mathrm{~m} ; 15 \mathrm{H}\right.$, Aromaten-H). - $\left.{ }^{13} \mathrm{C}-\mathrm{NMR}(\mathrm{CD}) \mathrm{Cl}_{3}\right)$ : $\delta=35.3$ (t; C-4), 48.7, 51.7 (2 d; C-1, C-5), 84.9 (s; C-6), 126.9, 127.1, 127.3, 127.0, 128.2, 1129.2 (Phenyl-C), 130.4, 132.8 (2 d; C-2, C-3), 136.5, 138.6, 140.0, 142.3, 146.3 (5 s; $3 \mathrm{C}_{\text {ipso, }}$ C-7, C-8). - MS (70 eV): $m / e=350\left(51 \%, \mathrm{M}^{+}\right), 349(24), 332$ (6), 284 (41), 283 (62), 245 (77), 231 (54), 206 (66), 178 (100), 167 (73), 165 (62), 152 (42), 105 (56).

trans-4-(Triphenylpropadienyl)-2-cyclopenten-1-ol (21): Farbloses, mikrokristallines Matterial mit Schmp. $132.5-133.5^{\circ} \mathrm{C}$ (Ether). - IR (KBr): $3400(\mathrm{OH}), 3050,3020,2930,2960,2860(\mathrm{CH})$, 1480,1590 (Aromatengerüst), 685, 760, 1040, 1440, 730, 1020,1110 cm-1. - ${ }^{1} \mathrm{H}-\mathrm{NMR}\left(\mathrm{CDCl}_{3}\right)$ : $\delta=1.78$ (s; $1 \mathrm{H}, \mathrm{OH}), 2.28$ (br. t, AB-Teil eines ABXY-Spektrums, scheinbare Kopplumg = $5 \mathrm{~Hz} ; 2 \mathrm{H}, 5 \mathrm{a}-\mathrm{H}, 5 \mathrm{~b}-\mathrm{H}$ ), 4.27 (mc; $1 \mathrm{H}, 4 \mathrm{H}), 5.00$ (mc; $1 \mathrm{H}, 1-\mathrm{H}), 6.05$ (mc; 2H, 2-H, 3-H), 7.33 (mc; $15 \mathrm{H}$, Aromaten-H). $-{ }^{13} \mathrm{C}-\mathrm{NMR}\left(\mathrm{CDCl}_{3}\right): \delta=41.2$ (t; C-5), 44.7 (d; C-4), 77.0 (d; C-1), 112.8, 114.0 (2 s; C-6, C-8), 126.3, 127.2, 127.3, 128.0, 128.2, 128.4, 128.6 (Aromaten-C), 133.9, 137.4 ( $2 \mathrm{~d} ; \mathrm{C}-2, \mathrm{C}-3), 135.6\left(\mathrm{~s} ; \mathrm{C}_{\mathrm{ipso}}\right), 136.5\left(\mathrm{~s} ; 2 \mathrm{C}_{\mathrm{ipso}}\right), 205.5$ (s; C-7). - MS (70 eV): $\mathrm{m} / \mathrm{e}=350$ $\left(33 \%, M^{+}\right), 268(26), 267(100), 265(16), 167$ (22), $165(20)$.

$\begin{array}{rlllll}\mathrm{C}_{26} \mathrm{H}_{22} \mathrm{O} \text { (350.5) } & \text { Ber. C } 89.11 & \text { H } 6.33 & & & \\ \text { 16: Gef. C } 88.95 & \text { H } 6.11 & \text { 17: Gef. C } 89.04 \text { H } 6.17 \\ \text { 18: Gef. C } 89.11 & \text { H } 6.34 & \text { 19: Gef. C } 89.09 \text { H } 6.33 \\ \text { 20: Gef. C } 86.13 & \text { H } 6.01 & \text { 21: Gef. C } 89.04 \text { H } 6.04\end{array}$

\section{Lanthaniden-Verschiebungsexperimente an den Alkoholen 16-21}

Käufliches Europium(III)-1,1,1,2,2,3,3-heptafluor-7,7-dimethyl-4,6-octandionat $=\operatorname{Eu}(\text { fod })_{3}$ wurde vor den Messungen $6 \mathrm{~h}$ bei $80^{\circ} \mathrm{C}$ im Ölpumpenvakuum getrocknet, in $\mathrm{CDCl}_{3}$ gelöst und mit Hilfe einer Mikroliterspritze zur Lösung des Substrats in $\mathrm{CDCl}_{3}$ gegeben. Tab. 3 verdeutlicht am Beispiel von 19, wie sich die chemischen Verschiebungen der einzelnen Protonen dabei ändern. Die graphische Darstellung in Abb. 2 zeigt eine lineare Abhängigkeit der induzierten chemischen Verschiebung von der Konzentration an Verschiebungsreagenz. Der Verschiebungsgradient $\Delta=\Delta \delta / \Delta\left(\mathrm{mol} \mathrm{Eu}(\mathrm{fod})_{3} / \mathrm{mol}\right.$ Alkohol) wurde als Steigung dieser Geraden durch lineare Regressionsanalyse ermittelt und findet sich mit den entsprechenden Daten für die anderen Alkohole in Tab. 4.

Nach McConnell und Robertson $\left.{ }^{6}\right)$ läßt sich der Verschiebungsgradient $\Delta_{i}$ nach $\mathrm{Gl}$. (1) berechnen:

$$
\Delta_{i}=\frac{K\left(3 \cos ^{2} \Theta-1\right)}{r_{i}^{3}}
$$

$r_{i}$ bedeutet hierin den Abstand zwischen dem betrachteten Proton $\mathrm{H}_{i}$ und dem Europium-Ion, $\Theta$ den Winkel zwischen dem Abstandsvektor $r_{i}$ und der Bindung funktionelle Gruppe-Eu ${ }^{3+} . K$ ist eine molekülspezifische, von der Geometrie und der Art der funktionellen Gruppe abhängige Konstante.

Um $r_{i}$ und $\Theta$ durch Ausmessen an Dreiding-Molekülmodellen bestimmen zu können, wählten wir in Analogie zu den Arbeiten von Crumrine ${ }^{13)}$ und Wilt $^{8)}$ den $\mathrm{O}-\mathrm{Eu}^{3+}$-Abstand zu $3.0 \AA$ und den Winkel C-O-Eu zu $130^{\circ}$. Weitere geometrische Annahmen sind bei den einzelnen Molekülen in Tab. 4 vermerkt. Mit Hilfe von $r_{i}$ und $\Theta$ berechneten wir für alle nichtaromatischen Protonen eines Moleküls die Größe $\Delta_{i} / K$ und bestimmten schließlich $K$ in der Weise, daß sich eine optimale Übereinstimmung zwischen den berechneten und experimentellen Werten $\Delta$ ergab. Die aus

Chem. Ber. 118 (1985) 
Tab. 4 hervorgehende weitgehende Korrelation von $\Delta_{\exp }$ und $\Delta_{\text {ber }}$ bestătigt die oben getroffenen Strukiturzuordnungen.

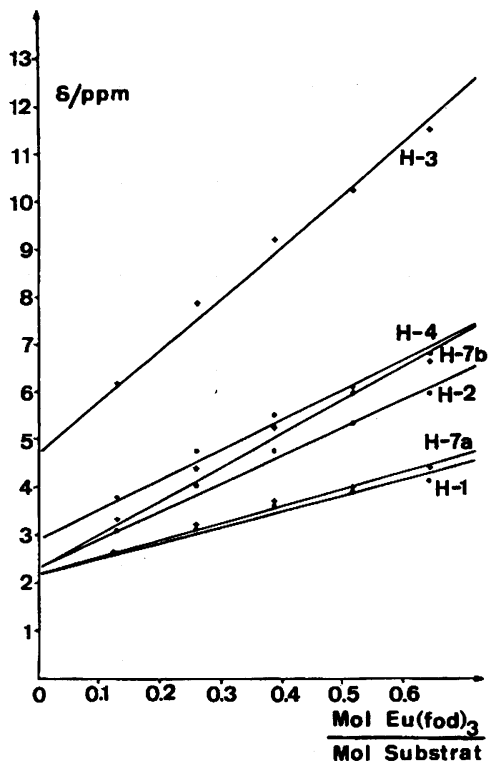

Abb. 2. ${ }^{1} \mathrm{H}$-Chemische Verschiebungen der Protonen des 5-(Diphenylmethylen)-6-phenyltricyclo$\left[2.2 .1 .0^{2,6}\right]$ heptan-exo-3-ols (19) in Abhängigkeit von zugegebenem Eu(fod) ${ }_{3}$-Verschiebungsreagenz

Tab. 3. ${ }^{1} \mathrm{H}$-Chemische Verschiebungen von 19 in Abhängigkeit von zugesetztem Eu(fod) ${ }_{3}$-Verschiebungsreagenz

\begin{tabular}{ccccccc}
\hline Eu(fod) 3 & $1-\mathrm{H}$ & $2-\mathrm{H}$ & $3-\mathrm{H}$ & $4-\mathrm{H}$ & $7 \mathrm{a}-\mathrm{H}$ & $7 \mathrm{~b}-\mathrm{H}$ \\
\cline { 1 - 4 } Substrat & & & & & & \\
\hline 0 & 2.05 & 2.05 & 4.32 & 2.70 & 2.05 & 2.05 \\
0.13 & 2.62 & 3.12 & 6.20 & 3.78 & 2.62 & 3.35 \\
0.26 & 3.13 & 4.03 & 7.88 & 4.75 & 3.23 & 4.42 \\
0.39 & 3.57 & 4.75 & 9.19 & 5.50 & 3.66 & 5.26 \\
0.52 & 3.89 & 5.32 & 10.25 & 6.08 & 4.00 & 5.97 \\
0.65 & 4.23 & 5.96 & 11.45 & 6.78 & 4.39 & 6.68 \\
\hline
\end{tabular}

\section{Umlagerungsreaktionen von 27}

$\alpha, \alpha, 3-T r i p h e n y l-2,5-n o r b o r n a d i e n-2-m e t h a n o l ~(27)$ : In einem 500-ml-Dreihalskolben wurden unter Stickstoffschutz $2.28 \mathrm{~g}(0.328 \mathrm{~mol}) \mathrm{Lithium}$ in $40 \mathrm{ml}$ absol. Ether vorgelegt. Innerhalb $1 \mathrm{~h}$ wurden $23.3 \mathrm{~g}(0.148 \mathrm{~mol})$ Brombenzol in $50 \mathrm{ml}$ absol. Ether unter Rühren so zugetropft, daß die Reaktionsmischung mäßig siedete. Nach dem Zutropfen wurde noch $1 \mathrm{~h}$ unter Rückfluß gekocht und dann das überschüssige Lithium mit einer Pinzette entfernt. Dann tropfte man $14.0 \mathrm{~g}(0.0619$ mol) 2614) in $50 \mathrm{ml}$ absol. Ether innerhalb von $2 \mathrm{~h}$ zu und rührte noch weitere $2 \mathrm{~h}$ bei Raumtemp. Es wurde mit $200 \mathrm{ml}$ Eiswasser hydrolysiert, die organische Phase abgetrennt und die waßßrige Phase mit $50 \mathrm{ml}$ Ether extrahiert. Die vereinigten Etherphasen wurden mit $50 \mathrm{ml}$ Wasser gewaschen und über $\mathrm{CaCl}_{2}$ getrocknet. Nach dem Abziehen des Ethers blieb ein kristalliner Rückstand, aus dem nach Umkristallisieren aus Ether $13.4 \mathrm{~g} \mathrm{(61 \% )} \mathrm{blaßgelbe} \mathrm{Prismen} \mathrm{vom} \mathrm{Schmp.} 99^{\circ} \mathrm{C}$ er-

Chem. Ber. 118 (1985) 
Tab. 4. Eu(fod) ${ }_{3}$-induzierte chemische Verschiebungen

\begin{tabular}{|c|c|c|c|c|c|c|c|}
\hline & $\delta_{0}^{\text {a) }}$ & $\Delta_{\exp }^{16}{ }^{b)}$ & $\Delta_{\text {ber }}{ }^{c)}$ & & $\delta_{0}^{a)}$ & $\Delta_{\exp }^{17}$ & $\Delta_{\text {ber }}{ }^{d, e)}$ \\
\hline $\begin{array}{l}1-\mathrm{H} \\
2-\mathrm{H} \\
3-\mathrm{H} \\
4 \mathrm{a}-\mathrm{H} \\
4 \mathrm{~b}-\mathrm{H} \\
5-\mathrm{H}\end{array}$ & $\begin{array}{l}4.27 \\
5.85 \\
5.85 \\
3.09 \\
2.47 \\
3.28\end{array}$ & $\begin{array}{l}0.26 \\
0.04 \\
0.04 \\
0.26 \\
0.07 \\
0.41\end{array}$ & & $\begin{array}{l}1-\mathrm{H} \\
2-\mathrm{H} \\
3-\mathrm{H} \\
4 \mathrm{a}-\mathrm{H} \\
4 \mathrm{~b}-\mathrm{H} \\
5-\mathrm{H}\end{array}$ & $\begin{array}{l}4.90 \\
5.99 \\
5.93 \\
2.43 \\
2.92 \\
3.36\end{array}$ & $\begin{array}{r}11.10 \\
2.50 \\
2.57 \\
2.43 \\
3.34 \\
7.55\end{array}$ & $\begin{array}{r}10.66 \\
2.24 \\
2.27 \\
2.94 \\
4.44 \\
7.13\end{array}$ \\
\hline & $\delta_{0}^{a)}$ & $\Delta_{\exp }^{18}$ & $\left.\Delta_{\text {ber }} d, f\right)$ & & $\delta_{0}^{a)}$ & $\Delta_{\exp }^{19}$ & $\left.\Delta_{\text {ber }}{ }^{d, g}\right)$ \\
\hline $\begin{array}{l}1-\mathrm{H} \\
4-\mathrm{H} \\
5-\mathrm{H} \\
6-\mathrm{H} \\
7 \mathrm{a}-\mathrm{H} \\
7 \mathrm{~b}-\mathrm{H}\end{array}$ & $\begin{array}{l}3.30 \\
3.73 \\
6.56 \\
5.89 \\
1.87 \\
2.62\end{array}$ & $\begin{array}{l}2.19 \\
0.62 \\
0.54 \\
0.65 \\
0.58 \\
1.97\end{array}$ & $\begin{array}{l}2.31 \\
0.96 \\
0.55 \\
0.52 \\
0.85 \\
1.78\end{array}$ & $\begin{array}{l}1-\mathrm{H} \\
2-\mathrm{H} \\
3-\mathrm{H} \\
4-\mathrm{H} \\
7 \mathrm{a}-\mathrm{H} \\
7 \mathrm{~b}-\mathrm{H}\end{array}$ & $\begin{array}{l}2.17 \\
2.37 \\
4.71 \\
2.92 \\
2.16 \\
2.26\end{array}$ & $\begin{array}{r}3.36 \\
5.80 \\
10.88 \\
6.21 \\
3.60 \\
7.21\end{array}$ & $\begin{array}{r}3.64 \\
5.73 \\
14.82 \\
6.03 \\
3.72 \\
6.39\end{array}$ \\
\hline & $\delta_{0}^{\text {a) }}$ & $\Delta_{\exp }^{20}$ & $\Delta_{\text {ber }}{ }^{d, h)}$ & & $\delta_{0}^{a)}$ & $\Delta_{\exp }^{21}{ }^{b)}$ & $\Delta_{\text {ber }}{ }^{d, i)}$ \\
\hline $\begin{array}{l}1-\mathrm{H} \\
2-\mathrm{H} \\
3-\mathrm{H} \\
4 \mathrm{a}-\mathrm{H} \\
4 \mathrm{~b}-\mathrm{H} \\
5-\mathrm{H}\end{array}$ & $\begin{array}{l}4.16 \\
5.69 \\
5.40 \\
1.65 \\
2.23 \\
2.83\end{array}$ & $\begin{array}{l}1.87 \\
1.27 \\
1.02 \\
2.03 \\
0.97 \\
5.75\end{array}$ & $\begin{array}{l}1.92 \\
1.10 \\
0.87 \\
3.44 \\
0.76 \\
6.87\end{array}$ & $\begin{array}{l}1-\mathrm{H} \\
2-\mathrm{H} \\
3-\mathrm{H} \\
4-\mathrm{H} \\
5 \mathrm{a}-\mathrm{H} \\
5 \mathrm{~b}-\mathrm{H}\end{array}$ & $\begin{array}{l}5.08 \\
6.08 \\
6.08 \\
4.03 \\
2.38 \\
2.28\end{array}$ & $\begin{array}{r}17.01 \\
8.88 \\
6.53 \\
6.05 \\
5.45 \\
11.33\end{array}$ & $\begin{array}{r}17.91 \\
9.20 \\
4.21 \\
5.49 \\
6.58 \\
11.52\end{array}$ \\
\hline
\end{tabular}

a) Auf (mol Verschiebungsreagens $/ \mathrm{mol}$ Alkohol) $=0$ extrapolierte chemische Verschiebungen. -

b) $\Delta_{\text {exp }}=\Delta \delta / \Delta$ (mol Verschiebungsreagens/mol Alkohol). - c) Wegen der geringen Verschiebung ist eine Korrelation zwischen $\Delta_{\text {exp }}$ und $\Delta_{\text {ber }}$ nicht aussagekräftig. - d) $K\left(3 \cos ^{2} \Theta-1\right) / r_{i}^{3}$ (McConnell, Lit. $\left.{ }^{6}\right)$. - e) $K=637$; Interplanarwinkel C-5, C-1, O, Eu $=180^{\circ}$. - ी $K=195$; Interplanarwinkel C-3, C-2, O, Eu $\left.=180^{\circ} .-\mathrm{g}\right) K=534$; Interplanarwinkel 3-H, C-3, O, Eu = $0^{\circ}$. - h) $K=727$; Interplanarwinkel $\mathrm{C}-5, \mathrm{C}-6, \mathrm{O}, \mathrm{Eu}=0^{\circ} .-$ i) $K=843$; Interplanarwinkel 1-H, $\mathrm{C}-1, \mathrm{O}, \mathrm{Eu}=0^{\circ}$.

halten wurden. - IR (KBr): $3560(\mathrm{OH}), 2960,3020,2930,3050,2860(\mathrm{CH}), 1590(\mathrm{C}=\mathrm{C}), 1440$, 1480, 1550 (Aromatengerüst), 695, 765, 1000, $990 \mathrm{~cm}^{-1}$. - ${ }^{1} \mathrm{H}-\mathrm{NMR}\left(\mathrm{CDCl}_{3}\right): \delta=1.87,2.37$ (2 br. d, $\left.J_{7 \mathrm{a}, 7 \mathrm{~b}}=6.0 \mathrm{~Hz} ; 2 \mathrm{H}, 7 \mathrm{a}-\mathrm{H}, 7 \mathrm{~b}-\mathrm{H}\right), 2.73$ (br. s; $\left.1 \mathrm{H}, \mathrm{OH}\right), 3.32,3.65(2 \mathrm{mc} ; 2 \mathrm{H}, 1-\mathrm{H}$, $4-\mathrm{H}), 6.42\left(\mathrm{dd}, J_{5,6}=4.8 \mathrm{~Hz}, J_{1,6}=1.6 \mathrm{~Hz} ; 1 \mathrm{H}, 6-\mathrm{H}\right), 6.85\left(\mathrm{dd}, J_{5,6}=4.8 \mathrm{~Hz}, J_{4,5}=2.4 \mathrm{~Hz}\right.$; $1 \mathrm{H}, 5-\mathrm{H}), 7.09,7.23\left(2 \mathrm{mc} ; 15 \mathrm{H}\right.$, Aromaten-H). $-{ }^{13} \mathrm{C}-\mathrm{NMR}\left(\mathrm{CDCl}_{3}\right): \delta=55.5,58.6(2 \mathrm{~d} ; \mathrm{C}-1$, $\mathrm{C}-4), 69.3$ (t; C-7), 81.5 (s; C-OH), 126.6, 127.0, 127.3, 127.6, 127.9 (Aromaten-C), 141.0, 142.9 (2 d; C-5, C-6), 138.1, 144.4, 146.0, 148.5, 152.9 (5 s; C-5, C-6, $3 \mathrm{C}_{\text {ipso }}$ ). - MS (70 eV): $m / e=350\left(58 \%, M^{+}\right), 332$ (5), 284 (24), 245 (12), 207 (26), 183 (35), 167 (11), 133 (49), $105(100)$.

$$
\mathrm{C}_{26} \mathrm{H}_{22} \mathrm{O}(350.5) \text { Ber. C 89.11 H 6.33 Gef. C } 89.07 \mathrm{H} 6.39
$$

Umlagerung von $27 z u$ 18: $200 \mathrm{mg}(0.57 \mathrm{mmol}) 27$ in $1 \mathrm{ml}$ THF wurden mit $20 \mathrm{ml} 50$ proz. Ameisensäure versetzt. Nach $24 \mathrm{~h}$ bei Raumtemp. wurden $30 \mathrm{ml}$ Ether zugesetzt, die Ameisensäure mit dest. Wasser (viermal $50 \mathrm{ml}$ ) ausgewaschen und die organische Phase über $\mathrm{K}_{2} \mathrm{CO}_{3} / \mathrm{CaCl}_{2}$ getrocknet. Nach Abziehen des Ethers erhielt man 195 mg (97.5\%) gelbes Öl, das als spektroskopisch reines 18 identifiziert werden konnte. Durch Zugabe von wenig Ethanol kristallisierte das Öl, es wurden $150 \mathrm{mg} 18$ isoliert. Bei $60^{\circ} \mathrm{C}$ war die Umlagerung von $27 \mathrm{zu} 18$ schon nach $2 \mathrm{~h}$ beendet. 
Umlagerung von $27 z u$ 19: $200 \mathrm{mg}(0.57 \mathrm{mmol}) 27$ in $5 \mathrm{ml}$ Dioxan wurden mit einer Lösung von $1 \mathrm{~g} p$-Toluolsulfonsäure in $1.5 \mathrm{ml}$ dest. Wasser versetzt und $3 \mathrm{~h}$ auf $70^{\circ} \mathrm{C}$ erwärmt. Dann wurden $40 \mathrm{ml}$ Ether zugegeben und die $p$-Toluolsulfonsäure mit $\mathrm{Na}_{2} \mathrm{CO}_{3}$-Lösung ausgewaschen. Die Etherphase wurde nochmals mit dest. Wasser (dreimal $10 \mathrm{ml}$ ) gewaschen und über $\mathrm{Na}_{2} \mathrm{SO}_{4}$ getrocknet. Nach Abdampfen des Ethers blieben $190 \mathrm{mg}$ (95\%) kristalliner Rückstand, der als spektroskopisch reines 19 identifiziert wurde.

1) H. Mayr und I. K. Halberstadt-Kausch, Chem. Ber. 115, 3479 (1982).

2) $H$. Mayr und $E$. Bäuml, Tetrahedron Lett. 24, 357 (1983).

3) Vgl. hierzu 3a) H. Mayr, Angew. Chem. 93, 202 (1981); Angew. Chem., Int. Ed. Engl. 20, 184 (1981), und 3b) $H$. Mayr und $W$. Striepe, J. Org. Chem. 48, 1159 (1983).

4) Zur Rolle des Silber-Gegenions vgl. H. M. R. Hoffmann, G. F. P. Kernaghan und G. Greenwood, J. Chem. Soc. B 1971, 2257.

5) $K$. H. Meyer und $K$. Schuster, Ber. Dtsch. Chem. Ges. 55, 819 (1922).

6) H. M. McConnell und R. E. Robertson, J. Chem. Phys. 29, 1361 (1958).

7) $A$. Chollet und P. Vogel, Helv. Chim. Acta 61, 732 (1978).

8) J. W. Wilt und $R$. Niinemae, J. Org. Chem. 45, 5402 (1980).

9) E. Bäuml und H. Mayr, Chem. Ber. 118, 694 (1985), nachstehend.

10) Der erste Schritt dieser Reaktionssequenz entspricht einem für Vinylkation-Cycloadditionen vorgeschlagenen Mechanismus: $H$. U. Wagner und $R$. Gompper, Tetrahedron Lett. 1971, 4061 .

11) T. L. Jacobs und D. M. Fenton, J. Org. Chem. 30, 1808 (1965).

12) $K$. Heß und W. Weltzien, Ber. Dtsch. Chem. Ges. 54, 2511 (1921).

13) D. S. Crumrine und H.-H. B. Yen, J. Org. Chem. 41, 1273 (1976).

14) E. Bäuml und H. Mayr, J. Org. Chem. 48, 2600 (1983). 